\title{
Food Acculturation Drives Dietary Differences among Mexicans, Mexican Americans, and Non-Hispanic Whites ${ }^{1-3}$
}

\author{
Carolina Batis, ${ }^{4}$ Lucia Hernandez-Barrera, ${ }^{5}$ Simon Barquera, ${ }^{5}$ Juan A. Rivera, ${ }^{5}$ and Barry M. Popkin ${ }^{4 *}$ \\ ${ }^{4}$ Department of Nutrition, University of North Carolina, Chapel Hill, NC; and ${ }^{5}$ Nutrition and Health Research Center, National Institute \\ of Public Health, Cuernavaca, Mexico
}

\begin{abstract}
Our aim was to examine the effects of food acculturation on Mexican Americans' (MA) diets, taking the Mexican diet as reference. We used nationally representative samples of children (2-11 y) and female adolescents and adults (12-49 y) from the Mexican National Nutrition Survey 1999 and NHANES 1999-2006 to compare the diets of Mexicans $(n=5678)$, MA born in Mexico (MAMX) ( $n=1488$ ), MA born in the United States (MAUS) ( $n=3654$ ), and non-Hispanic white Americans (NH-White) $(n=5473)$. One 24-h diet recall was used to examine the percentage consuming and percentage energy consumed from selected food groups. Most of the food groups analyzed displayed a fairly linear increase or decrease in percent energy/capita intake in this order: Mexican, MAMX, MAUS, NH-White. However, few significant differences were observed among the US subpopulations, especially among MAUS and NH-Whites. Overall, compared to Mexicans, the US subpopulations had greater intakes of saturated fat, sugar, dessert and salty snacks, pizza and French fries, low-fat meat and fish, high-fiber bread, and low-fat milk, as well as decreased intakes of corn tortillas, low-fiber bread, high-fat milk, and Mexican fast food. Furthermore, the patterns were similar in all age groups. Although we found a mix of positive and negative aspects of food acculturation, the overall proportion of energy obtained from unhealthy foods was higher among the US subpopulations. Our findings indicate that within one generation in the US, the influence of the Mexican diet is almost lost. In addition, our results reinforce the need to discourage critical unhealthful components of the American diet among MA. J. Nutr. 0: 1898-1906, 2011.
\end{abstract}

\section{Introduction}

In 2010, Hispanics in the United States numbered over 50.5 million, or about 1 in 6 people (1) and, by 2050, the ratio is expected to reach 1 Hispanic in 4 people in the US. Of the Hispanics in the US in 2002, 66.9\% were of Mexican origin $(2,3)$. Despite higher levels of poverty, lower educational attainment, and other socioeconomic disadvantages, Hispanic immigrants do better than, or comparable to, non-Hispanic Whites (NH-Whites) ${ }^{6}$ in overall mortality as well as cardiovascular disease and malignant neoplasms mortality (4). A proposed explanation for this so-called Hispanic health paradox

\footnotetext{
${ }^{1}$ Supported by the Consejo Nacional de Ciencia y Tecnologia (grant no. 212018), the Robert Wood Johnson Foundation (grant no. 67506), and the NIH (R01 HL104580).

2 Author disclosures: C. Batis, L. Hernandez-Barrera, S. Barquera, J. A. Rivera, and B. M. Popkin, no conflicts of interest.

${ }^{3}$ Supplemental Tables 1-4 are available as Online Supporting Material with the online posting of this paper at http://jn.nutrition.org.

${ }^{6}$ Abbreviations used: FNDDS, Food and Nutrient Database for Dietary Studies; MA, Mexican American; MAMX, Mexican American born in Mexico; MAUS, Mexican American born in United States; MNS, Mexican Nutrition Survey; NH-White, non-Hispanic white; USDA-SR, USDA National Nutrient Database for Standard Reference.

* To whom correspondence should be addressed. E-mail: popkin@unc.edu.
}

suggests that the original culture might be protective among new immigrants; however, as they undergo the acculturation process and learn or adopt aspects of the US culture, those protective behaviors are lost $(4,5)$. The adoption of the US diet, here called "food acculturation," or other obesity-related behaviors seems to be of particular importance when explaining the health paradox. Studies using country of birth, generational status, and length of time in the US as a proxy of acculturation have found that the more acculturated Hispanics have higher BMI than those who were less acculturated (6-8). However, overall, there is a higher prevalence of obesity among Mexican Americans (MA) than among NH-Whites (9).

The health paradox becomes more complex when we consider that the original MA immigrants' culture is also undergoing changes. Mexico is experiencing an epidemiological and nutritional transition and its population is approaching the cardio-metabolic profile observed in the US. Overweight and obesity increased dramatically during the last 2 decades. Fat, sugar, refined carbohydrate, and soda intake have also increased among the Mexican population as has an array of cardiovascular problems (10-13).

Several studies have described the role of acculturation on the MA diet. Both beneficial and deleterious changes have been reported, but, in general, most research notes an overall 
deleterious net effect on the MA diet (14-17). These studies have compared the diets of MA in the US with different levels of acculturation using the less acculturated group as the reference. However, strictly speaking, all MA groups have already undergone at least some degree of acculturation. All are self-selected immigrants and all have been directly exposed to the US environment. To fill this important gap and better understand the effects of food acculturation on the quality of the MA diet, we used a nonacculturated group as the reference, namely, the Mexican population.

This study used data from Mexico and the US. The foods in each data set were linked to systematically group identical foods in both surveys. This allowed us to compare the diets of persons residing in Mexico with 3 subpopulations residing in the US: Mexican Americans born in Mexico (MAMX), Mexican Americans born in the US (MAUS), and NH-White. Our aims in comparing these 4 subpopulations with different degrees of exposure to the US environment were, first, to identify the beneficial or deleterious effects of adopting a US diet, using the Mexican diet as reference, and, second, to assess the influence of the Mexican diet relative to the influence of the American diet on the MAMX and MAUS subpopulations. This information provides insight into how to address and intervene in the nutrition-related problems of the MA population. In addition, we examined a number of key age-gender groupings, including children of both genders, female adolescents, and adult women.

\section{Methods}

Participants. This analysis used secondary data from nationally representative samples from both Mexico and the US. For Mexico, we used data from the MNS 1999, conducted by the Mexican National Institute of Public Health between October 1998 and March 1999. For this survey, a probabilistic sample representative of urban and rural areas was selected. A detailed description of the sampling strategy and survey methodology was previously published (18). Due to budget restrictions, only information for women ( $12-49 \mathrm{y}$ old $)$ and children (2-11 y old) was collected and dietary information was collected from a nationally representative subsample of the MNS 1999. For this analysis, we included all women and children with complete dietary data.

For the US, we used data from NHANES, which includes a representative sample of the US population of all ages. People $\geq 60$ y old, African Americans, and Hispanics are oversampled. To have an adequate sample size of MA, we combined data for 8 y of surveys (1999-2006). However, before doing so, we examined the differences between the 4 surveys (1999-2000, 2001-2002, 2003-2004, and 2005-2006) among the entire population included in this analysis by age-group and found no significant differences $(P<0.01)$ for our main measures of interest. Detailed information about each survey and its sampling design has been published elsewhere (19). For this analysis, we included women (12-49 y old) and children (2-11 y old) and created comparison groups among participants that identified themselves as MAMX, MAUS, and NHWhites. The Institutional Review Board of the National Institute in Mexico approved data collection and provided expedited approval for use of these secondary data for this article. For NHANES, written informed consent was obtained from all participants; by utilizing secondary NAHNES data, we were exempt from institutional review board monitoring for this paper.

Dietary intake, food composition tables, and food groups. Dietary intake analysis was based on a single 24-h dietary recall from each survey, whereby participants reported all foods and beverages consumed during the previous day. For MNS 1999, one 24-h recall was performed at the household of the participant. Trained personnel applied the questionnaires and converted each reported preparation into grams or milliliters of individual foods; however, no standardized probing method (i.e. 5-pass method) was used in this survey. For NHANES, the 24-h recalls were conducted in English or Spanish by dietary interviewers with a computer-assisted automated data collection system. Beginning in 2002, the collection system changed to a multiple 5-pass method, which covers essentially the same questions used in 1999-2001 but in a slightly different order $(20,21)$. In addition, after 2002, participants were asked to complete a second 24-h dietary recall interview by telephone. To maintain consistency among all surveys, we included only the first 24-h recall from each in this analysis.

The NHANES nutrient intake was estimated using the USDA FNDDS, which is based on the USDA-SR. The main differences between the databases are that the FNDDS does not have missing values and includes a larger variety of portion sizes and food mixtures. The NHANES 1999-2000 survey used a different food composition table that does not include sugar, the USDA 1994-1998 Survey Nutrient Data Base (22); therefore, for these years, we used FNDDS 1.0 (NHANES 2001-2002). Eighty-one foods reported by this sample were not included in FNDDS 1.0. For these foods, the original food composition table was used and sugar content was imputed based on the carbohydrate:sugar ratios of similar foods.

MNS 1999 originally used a comprehensive nutrient composition database compiled from diverse references by the Nutrition and Health Research Center, National Institute of Public Health. This compilation was based on the USDA-SR for one-half of the foods (348 of 680 foods reported in our sample). For the present analysis, we revised this database to make it more comparable to the one used in NHANES. After an exhaustive review, food-by-food, we found that FNDDS was appropriate for 375 of the foods, because the energy content and/or the description of the food were similar in both food composition tables. We continued to use the initial compilation's source for the rest of the foods, which was the USDA-SR for 105 foods. The revised database still had 93 foods with sugar content missing, so we imputed these values based on the carbohydrate:sugar ratios of similar foods.

Diet comparisons were based on a food grouping system developed at The University of North Carolina-Chapel Hill (23). By using this system, we could summarize intake in a nutritionally and behaviorally meaningful way. The system was developed by further disaggregating the major food groups of the USDA by fat and fiber content. The version we used had 107 food groups and, for this study, we added 3 more groups: corn tortillas (including other corn products), wheat tortillas, and atole (corn- or other cereal-based Mexican beverages). We first performed the analysis using all groups. Then, we focused only on the food groups that were captured well by both surveys. In the MNS 1999, most of the intake was reported by ingredient and not by the food as prepared, whereas, in the NHANES, reporting the food as prepared was more common. Therefore, we observed, e.g., a higher consumption of oils, sugar, and flour and a lower consumption of hamburgers or other mixed dishes in the MNS 1999 compared to the NHANES. Because this discrepancy results from the way diet is reported and not from actual intake differences, we did not present information on these groups.

For adult and adolescent women, we wanted to capture the intake of sugar-sweetened beverages like coffee, tea, and fruit drinks. So, in both surveys, we aggregated the beverage ingredients that were recorded separately into a single food group. For example, if coffee, milk, and sugar were recorded as part of a single preparation, we combined that information into a single group called "coffee with milk and sugar." We also aggregated some foods groups that had similar nutritional or behavioral values. In addition, we do not present the food groups that comprised $<1 \%$ of the total energy intake per capita or food groups with noncaloric items consumed by $<5 \%$ of the participants in either subpopulation.

Statistical analysis. Both the MNS 1999 and NHANES are complex sample surveys; therefore, we analyzed each of them separately using the appropriate weighted survey commands to account for the study designs and sampling methods. Diets can differ between subpopulations with respect to the proportion of people consuming a specific food group and/ or the amount of that food group each person consumes. Therefore, for examination of dietary data patterns, we focused on 3 measures of a food group's intake: the percentage consuming each food group (percent 
consuming); the mean percentage of total energy from that food/ beverage group among consumers (percent energy/consumers); and, for the total sample, the mean percentage of total energy per capita (percent energy/capita). The last is a summary of the first 2 , because it is obtained by multiplying percent consuming by the percent energy/consumers. Data are presented as means or percent \pm SEM.

Age standardization based on census 2000 population data (10-y group distribution) was used for all adult women. Statistical comparisons were performed with a z-test using the SE computed for each mean or proportion with survey commands, as previously suggested (24). In this case, using the sample variance instead of the population variance was a fair approximation because of our sample sizes. To confirm this, we also computed the $t$ test when possible; e.g., when comparing groups coming from the same survey (NHANES), both the z-test and the $t$ test were concordant when identifying significant results (though results from $t$ test are not shown). We adjusted the $P$-value for multiple comparisons with Bonferroni correction. $P<0.01$ was considered significant. All analyses were conducted in Stata 11.1 (StataCorp).

\section{Results}

Demographics. A few important differences in demographic characteristics existed (Table 1). NH-White adult women were slightly older compared to all other subpopulations $(P<0.01)$. The proportion of adult women with less than a high school education was higher in the Mexican and MAMX subpopulations compared to the MAUS and NH-Whites. Among children (7-11 y old), the proportion of overweight or obese individuals (BMI for age $\geq 85$ th percentile) (25) was significantly lower in the Mexicans compared to all other subpopulations. However, among adult women, the proportion was significantly lower for NH-Whites compared to all other subpopulations.

Mexican vs. US diets: the positive and negative effects of food acculturation. Total energy intake was lower in Mexicans of all ages than in all other subpopulations $(P<0.01)$ (Table 1$)$, whereas there were no significant differences among the US subpopulations. Consequently, we present all dietary results as a percentage of energy/day (percent energy). Based on nutrients, Mexicans had a more suitable dietary profile, with a lower proportion of energy from saturated fat and sugar compared to all other subpopulations $(P<0.01)$.

With respect to beverages, among adult women, we observed a mix of positive and negative effects of acculturation to the US diet. The proportion of participants consuming coffee without sugar was higher among Mexicans compared to all others, whereas the proportion that consumed coffee with milk and sugar and tea with sugar was higher in the US subpopulations than in Mexicans. (Fig. 1; Supplemental Table 4). In the case of sodas, the proportion that consumed regular soda was significantly higher among MAMX and MAUS compared to Mexicans, and the proportion consuming diet sodas was significantly higher among MAUS and NH-White adult women compared to Mexicans and MAMX.

Although in all subpopulations a higher proportion preferred high-fat milk, a benefit of food acculturation was that the ratio of adult women drinking high-fat milk to those drinking low-fat milk was lower in the US subpopulations (139.4 among Mexicans, 5.2 among MAMX, 3.9 among MAUS, and 1.1 among NH-Whites). The consumption of milk with chocolate did not differ, and the proportion of adult women consuming atole was higher in the Mexican population compared to all other subpopulations $(P<0.01)$.

Similar patterns across subpopulations were found in female adolescents and children (Supplemental Tables 1-3). As ex- pected, a generally lower proportion of adolescents and children consumed coffee and tea and a higher proportion consumed milk and soda compared to adult women. Remarkably, considerably more Mexican children consumed coffee (30\% of 7-11 y olds; $24 \%$ of $2-6$ y olds) compared to US children (1-6\%).

With respect to food groups, we also noticed a mix of positive and negative adoptions (Table 2). Considering only food groups with large differences in percent energy/capita ( $\geq 4 \%$ difference between at least 2 subpopulations) among adult women (Fig. 2), the positive aspects of acculturation included a higher intake of low-fat meat and fish and high-fiber bread and a lower intake of low-fiber bread and Mexican fast food among the US subpopulations compared to Mexicans. On the negative side of acculturation to the US diet, we observed that consumption of dessert and salty snacks as well as pizza and French fries was higher among the US subpopulations. Another important difference was that Mexicans had a remarkably greater consumption of corn tortillas.

The effects of acculturation in terms of percent energy/capita can be due to a difference in percent consuming, percent energy/ consumers, or both. In the case of medium- and high-fat meat and eggs, nuts and seeds, ready-to-eat cereal, Mexican fast food, and pizza and French fries, the percent energy/consumers was basically the same across all 4 subpopulations $(P>0.01)$ (Supplemental Table 4). This means that differences in percent energy/capita were due to differences in percent consuming. On the contrary, in the case of low-fiber bread, corn tortillas, and dessert and salty snacks, both the percent consuming and percent energy/consumers differed among subpopulations in the same direction as percent energy/capita. This means that, e.g., the higher percent energy/capita in corn tortilla consumption observed among Mexicans was due to both a higher percent consuming and a higher percent energy/consumers.

The general patterns in adult women were fairly similar to those among female adolescents and children. Nevertheless, the intakes (percent energy/capita) of ready-to-eat cereal, dessert and salty snacks, pizza, and French fries were higher in female adolescents and children compared to adult women; also, the intake of corn tortillas was lower in children 2-6 y old than in adult women (Supplemental Tables 1-4).

When we aggregated many food groups into healthy and unhealthy options (Fig. 3), we saw that the percent energy/capita of healthy foods was significantly higher among Mexicans compared to all other subpopulations if we included tortillas in this category. However, if we did not include tortillas as a healthy item, then the percent energy/capita of healthy foods was lower or similar among Mexicans compared to the US subpopulations. In the case of unhealthy foods, the percent energy/capita generally increased as we moved from Mexicans to NH-Whites. In addition, unhealthy foods provided a higher proportion of energy intake for children and female adolescents $(41-59 \%)$ than for adult women $(36-46 \%)$.

The influence of the Mexican diet on the MA population. When we ordered the data by degree of exposure to the US environment (Mexican, MAMX, MAUS, and NH-White), we noticed in most of the food groups and in the healthy or unhealthy categories, a linear pattern between the percent energy/capita and the subpopulation's degree of US exposure (Supplemental Tables 1-4; Fig. 2). This may reflect a decrease in the influence of the Mexican diet within MA generations. Important exceptions to this linear pattern were fruits and vegetables and fruit juices, in which MAMX had a significantly higher intake compared to all other subpopulations, especially among adult women. 
TABLE 1 Sample characteristics and mean daily nutrient intake of Mexicans (MNS 1999), MA, and NH-Whites (NHANES 1999-2006) ${ }^{1}$

\begin{tabular}{|c|c|c|c|c|}
\hline & Mexican & MAMX & MAUS & NH-White \\
\hline \multicolumn{5}{|l|}{ Children 2-6 y old } \\
\hline$n$ & 1507 & 103 & 1146 & 1158 \\
\hline Male, $\%$ & $53.2 \pm 2.2$ & $45.1 \pm 5.7$ & $50.7 \pm 1.5$ & $50.1 \pm 1.6$ \\
\hline$\geq 5 y$ in the US, $\%$ & & $11.1 \pm 3.0$ & & \\
\hline Overweight or obese, ${ }^{2} \%$ & $26.8 \pm 1.7$ & $31.5 \pm 4.8$ & $26.8 \pm 1.6$ & $22.1 \pm 1.5$ \\
\hline \multicolumn{5}{|l|}{ Daily nutrient intake } \\
\hline Total energy, $\mathrm{kcal}$ & $1100 \pm 18^{b}$ & $1690 \pm 72^{\mathrm{a}}$ & $1650 \pm 27^{a}$ & $1660 \pm 22^{a}$ \\
\hline Fat, \% energy & $31.4 \pm 0.4$ & $30.7 \pm 0.7$ & $31.7 \pm 0.3$ & $32.0 \pm 0.3$ \\
\hline Saturated fat, $\%$ energy & $10.0 \pm 0.2^{b}$ & $11.3 \pm 0.4^{a}$ & $11.6 \pm 0.1^{a}$ & $11.7 \pm 0.1^{\mathrm{a}}$ \\
\hline Sugar, \% energy & $21.4 \pm 0.4^{b}$ & $30.6 \pm 1.1^{\mathrm{a}}$ & $30.1 \pm 0.3^{\mathrm{a}}$ & $30.4 \pm 0.3^{\mathrm{a}}$ \\
\hline Fiber, $g$ & $11.5 \pm 0.3^{\mathrm{ab}}$ & $14.3 \pm 1.1^{\mathrm{a}}$ & $11.7 \pm 0.3^{\mathrm{a}}$ & $10.5 \pm 0.2^{b}$ \\
\hline \multicolumn{5}{|l|}{ Children 7-11 y old } \\
\hline$n$ & 1761 & 185 & 911 & 881 \\
\hline Male, \% & $52.1 \pm 2.1$ & $53.6 \pm 4.0$ & $51.4 \pm 1.6$ & $51.9 \pm 2.0$ \\
\hline$\geq 5 \mathrm{y}$ in the US, $\%$ & & $49.9 \pm 5.0$ & & \\
\hline Overweight or obese, ${ }^{2} \%$ & $23.5 \pm 1.7^{\mathrm{c}}$ & $42.5 \pm 3.5^{\mathrm{ab}}$ & $45.3 \pm 1.8^{\mathrm{a}}$ & $34.4 \pm 2.2^{b}$ \\
\hline \multicolumn{5}{|l|}{ Daily nutrient intake } \\
\hline Total energy, $\mathrm{kcal}$ & $1470 \pm 23^{b}$ & $2100 \pm 45^{\mathrm{a}}$ & $2050 \pm 37^{a}$ & $2080 \pm 36^{a}$ \\
\hline Fat, \% energy & $29.4 \pm 0.4^{b}$ & $32.5 \pm 0.7^{\mathrm{a}}$ & $33.1 \pm 0.3^{\mathrm{a}}$ & $33.0 \pm 0.3^{\mathrm{a}}$ \\
\hline Saturated fat, $\%$ energy & $8.67 \pm 0.19^{b}$ & $11.6 \pm 0.4^{\mathrm{a}}$ & $11.7 \pm 0.1^{\mathrm{a}}$ & $11.7 \pm 0.1^{\mathrm{a}}$ \\
\hline Sugar, \% energy & $18.2 \pm 0.5^{b}$ & $27.1 \pm 0.8^{\mathrm{a}}$ & $27.5 \pm 0.3^{\mathrm{a}}$ & $28.7 \pm 0.4^{\mathrm{a}}$ \\
\hline Fiber, $g$ & $16.9 \pm 0.5^{\mathrm{a}}$ & $16.2 \pm 0.6^{\mathrm{ab}}$ & $14.2 \pm 0.4^{b}$ & $12.8 \pm 0.2^{c}$ \\
\hline \multicolumn{5}{|l|}{ Women $12-19$ y old } \\
\hline$n$ & 432 & 398 & 1,111 & 1,137 \\
\hline$\geq 10 y$ in the US, $\%$ & & $37.9 \pm 3.8$ & & \\
\hline Overweight or obese, ${ }^{3} \%$ & $26.5 \pm 2.9^{b}$ & $37.1 \pm 2.6^{\mathrm{ab}}$ & $42.7 \pm 2.0^{\mathrm{a}}$ & $31.0 \pm 1.7^{b}$ \\
\hline \multicolumn{5}{|l|}{ Daily nutrient intake } \\
\hline Total energy, $\mathrm{kcal}$ & $1640 \pm 44^{b}$ & $1930 \pm 46^{\mathrm{a}}$ & $2000 \pm 38^{a}$ & $1960 \pm 21^{a}$ \\
\hline Fat, \% energy & $29.6 \pm 0.9^{b}$ & $31.7 \pm 0.4^{\mathrm{ab}}$ & $33.0 \pm 0.4^{\mathrm{a}}$ & $32.6 \pm 0.3^{\mathrm{a}}$ \\
\hline Saturated fat, \% energy & $8.36 \pm 0.35^{b}$ & $10.7 \pm 0.2^{\mathrm{a}}$ & $11.1 \pm 0.1^{\mathrm{a}}$ & $11.3 \pm 0.1^{\mathrm{a}}$ \\
\hline Sugar, \% energy & $18.0 \pm 1.1^{b}$ & $27.3 \pm 0.5^{\mathrm{a}}$ & $27.4 \pm 0.4^{\mathrm{a}}$ & $28.6 \pm 0.4^{\mathrm{a}}$ \\
\hline Fiber, $g$ & $20.2 \pm 0.8^{\mathrm{a}}$ & $15.8 \pm 0.5^{b}$ & $13.1 \pm 0.3^{\mathrm{c}}$ & $12.0 \pm 0.2^{d}$ \\
\hline \multicolumn{5}{|l|}{ Women $20-49$ y old } \\
\hline$n$ & 1978 & 802 & 486 & 2297 \\
\hline Age, $y$ & $32.4 \pm 0.3^{b}$ & $33.0 \pm 0.4^{b}$ & $32.3 \pm 0.5^{b}$ & $35.2 \pm 0.2^{\mathrm{a}}$ \\
\hline$\geq 10 y$ in the US, $\%$ & & $51.8 \pm 2.3$ & & \\
\hline Less than high school, \% & $71.0 \pm 1.6^{\mathrm{a}}$ & $65.9 \pm 2.4^{\mathrm{a}}$ & $26.3 \pm 2.6^{b}$ & $11.4 \pm 1.1^{\mathrm{c}}$ \\
\hline Overweight or obese, ${ }^{3} \%$ & $66.4 \pm 1.7^{\mathrm{a}}$ & $69.7 \pm 2.1^{\mathrm{a}}$ & $71.5 \pm 2.4^{\mathrm{a}}$ & $53.0 \pm 1.7^{b}$ \\
\hline \multicolumn{5}{|l|}{ Daily nutrient intake } \\
\hline Total energy, $\mathrm{kcal}$ & $1420 \pm 20^{b}$ & $1920 \pm 35^{\mathrm{a}}$ & $2010 \pm 44^{a}$ & $1980 \pm 15^{a}$ \\
\hline Fat, \% energy & $29.7 \pm 0.4^{c}$ & $31.2 \pm 0.3^{b}$ & $34.6 \pm 0.5^{\mathrm{a}}$ & $33.8 \pm 0.2^{\mathrm{a}}$ \\
\hline Saturated fat, \% energy & $8.57 \pm 0.16^{c}$ & $10.4 \pm 0.2^{b}$ & $11.2 \pm 0.2^{\mathrm{ab}}$ & $11.2 \pm 0.1^{\mathrm{a}}$ \\
\hline Sugar, \% energy & $18.0 \pm 0.4^{b}$ & $25.2 \pm 0.4^{\mathrm{a}}$ & $23.7 \pm 0.5^{\mathrm{a}}$ & $24.7 \pm 0.4^{\mathrm{a}}$ \\
\hline Fiber, $g$ & $17.8 \pm 0.3^{\mathrm{a}}$ & $17.9 \pm 0.4^{\mathrm{a}}$ & $14.5 \pm 0.5^{b}$ & $13.9 \pm 0.3^{b}$ \\
\hline
\end{tabular}

\footnotetext{
${ }^{1}$ Values are means or percent \pm SEM. Values in a row with superscripts without a common letter differ, $P<0.01$ ( $z$-test with Bonferroni correction). MAMX, Mexican American born in Mexico; MAUS, Mexican American born in the US; NH-White, non-Hispanic white; MNS, Mexican Nutrition Survey.

${ }^{2} \mathrm{BMI}$ for age $\geq 85$ th percentile according to $2000 \mathrm{CDC}$ Growth Charts for the US (25).

${ }^{3} \mathrm{BMI} \geq 25 \mathrm{~kg} / \mathrm{m}^{2}$.
}

Another important finding was that the differences among subpopulations were mainly between Mexicans and all of the US subpopulations. We saw some differences between MAMX and MAUS or between MAMX and NH-Whites, but we did not find a difference $\geq 5 \%$ in percent energy/capita when we compared the MAUS to the NH-White subpopulation. This could be an indication that the influence of the Mexican diet relative to that of the American diet was almost absent in the MAUS subpop- ulation. In addition, for children and female adolescents, the influence of the Mexican diet might be even lower among the MAMX subpopulation. There were fewer significant differences when we compared MAMX to MAUS among children and female adolescents than among adult women. Also, fewer significant differences across all subpopulations were found among children 2-6 y old compared to other age groups when aggregating foods into healthy or unhealthy options. 


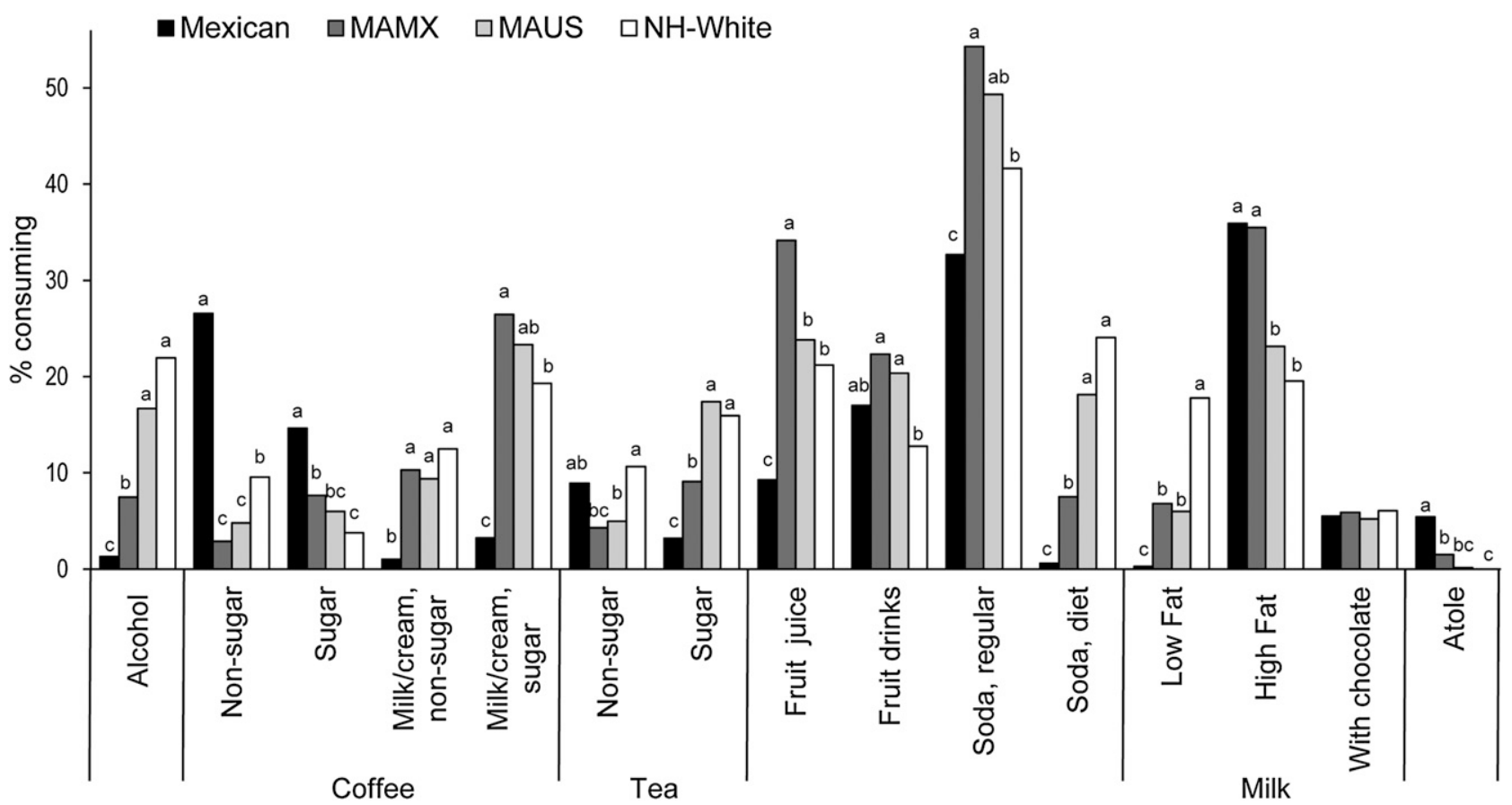

FIGURE 1 The percentage of Mexican, MA, and NH-White (NHANES 1999-2006) adult women consuming different types of beverages. $n=$ 1978 (Mexican), 802 (MAMX), 486 MAUS, or 2297 NH-White. Percentages without a common letter differ, $P<0.01$ (z-test with Bonferroni correction). MA, Mexican American; MAMX, Mexican American born in Mexico; MAUS, Mexican American born in the US; MNS, Mexican Nutrition Survey; NH-White, non-Hispanic white.

\section{Discussion}

By comparing not only US subpopulations of MA (MAMX, MAUS) but also Mexicans and NH-Whites, we saw the effects of exposure to the US environment and the influence of the Mexican diet relative to the American diet on MA food intake. Our main finding was that with food acculturation comes a mix of beneficial and deleterious diet adoptions. The beneficial dietary aspects we found included increased intake of low-fat meat and fish, high-fiber bread, and low-fat milk and decreased consumption of low-fiber bread, high-fat milk, and Mexican fast food. The deleterious aspects we found in our comparison included increased intake of saturated fat, sugar, dessert and salty snacks, pizza, and French fries. Overall, when we aggregated foods into healthy or unhealthy categories, the percent energy/capita of unhealthy foods was higher among the US subpopulations. The results for the healthy category depended on how tortillas were classified. The percent energy/capita of healthy foods was highest in Mexicans, relative to the US subpopulations, when tortillas were included and lower or similar in Mexicans when tortillas were excluded.

The largest difference was the much lower consumption of corn tortillas among MA compared to Mexicans. Corn tortillas are a low-fat, low-sodium, and high-fiber food that could be considered as healthy and, therefore, the lower intake of this food among MA may be classified as a negative effect of food acculturation. However, minimal research has focused on the healthfulness of tortillas. About $25 \%$ of the energy per capita among Mexicans came from corn tortillas; thus, if a reduction in tortilla intake promotes the inclusion of more varied and healthier foods in the diet, which we cannot determine, this reduction might be viewed as beneficial.

The main contribution of this study is that it included the diets of Mexican residents of ages comparable to the ages of the US subpopulations included. This allowed a clearer identifica- tion of the reasons for the similarities and differences between foreign-born and US-born MA. We could assess if these differences are related to influences of Mexican origin or to other unique features of these subpopulations. For most of the food groups, we found a fairly linear pattern between the percent energy/capita and the degree of exposure to the US environment. This means that the differences between MAMX and MAUS subpopulations could be seen as steps moving from the Mexican to the American diet. However, for some food groups, notably fruits and vegetables, fruit juices, and fruit drinks, the MAMX subpopulation had the highest percent energy/capita intake among adult women.

Fruit and vegetable intake has been repeatedly reported as higher among the MAMX subpopulation or first-generation Hispanics compared to the MAUS or second-generation Hispanics $(8,14,15,26,27)$. Yet our results indicate that the Mexican diet does not include a high intake of fruits and vegetables. A study that retrospectively evaluated the diets of women of Mexican descent living in California prior to and after their migration found that the consumption of vegetables and fruit juices increased after migration (28). The authors noted that, in a traditional Mexican diet, vegetables were used mainly as ingredients in the preparation of soups, rice, pasta, and meat, whereas salads and vegetable side dishes that were less common in Mexico were consumed more frequently after immigration to the US. This may explain why, in our results, the MAMX adult subpopulation consumed more fruits and vegetables than the Mexicans. However, it remains unclear why the MAMX subpopulation has a higher intake of fruits and vegetables compared to the MAUS subpopulation in this and previous studies.

By adding NH-Whites to our comparison, we were able to put into perspective the importance of the Mexican influence on MA populations. We can see that the diets of MAMX and MAUS had more in common with the American diet than with the Mexican 
TABLE 2 Mean difference in percentage of total energy per capita between Mexicans (MNS 1999) and other US subpopulations (NHANES 1999-2006) from selected food groups ${ }^{1}$

\begin{tabular}{|c|c|c|c|c|c|c|c|c|c|c|c|c|}
\hline \multirow[b]{3}{*}{ Food groups } & \multicolumn{12}{|c|}{ Mean difference in percent energy/capita between Mexicans and } \\
\hline & MAMX & MAUS & NH-White & MAMX & MAUS & NH-White & MAMX & MAUS & NH-White & MAMX & MAUS & NH-White \\
\hline & \multicolumn{3}{|c|}{ Children $2-6$ y old } & \multicolumn{3}{|c|}{ Children $7-11$ y old } & \multicolumn{3}{|c|}{ Women $12-19$ y old } & \multicolumn{3}{|c|}{ Women $20-49$ y old } \\
\hline \multicolumn{13}{|l|}{ Positive effects of food acculturation } \\
\hline \multicolumn{13}{|l|}{ Healthy foods with higher intake in the US } \\
\hline Low-fat milk ${ }^{2}$ & 0.8 & $0.9^{*}$ & $2.1^{*}$ & 0.7 & $0.6^{*}$ & $1.8^{*}$ & $0.5^{*}$ & $0.7^{*}$ & $1.4^{*}$ & $0.6^{*}$ & $0.4^{*}$ & $1.2^{*}$ \\
\hline Nuts and seeds & 0.1 & $0.5^{*}$ & $1.3^{*}$ & 0.4 & 0.5 & $0.9^{*}$ & -0.4 & -0.1 & 0.5 & $0.7^{*}$ & $1.1^{*}$ & $1.3^{*}$ \\
\hline High-fiber bread ${ }^{4}$ & $1.3^{*}$ & $1.9^{*}$ & $3.2^{*}$ & $2.1^{*}$ & $2.6^{*}$ & $3.7^{*}$ & $1.6^{*}$ & $2.7^{*}$ & $3.4^{*}$ & $1.4^{*}$ & $2.8^{*}$ & $4.2^{*}$ \\
\hline \multicolumn{13}{|l|}{ Unhealthy foods with lower intake in the US } \\
\hline High-fat milk² & -1.2 & -0.5 & $-3.3^{*}$ & 0.8 & 0.7 & $-1.9^{*}$ & 0.7 & 0.0 & -0.4 & -0.4 & $-2.1^{*}$ & $-2.5^{*}$ \\
\hline Medium-and high-fat meats and eggs ${ }^{3}$ & -0.6 & -1.3 & -2.0 & 0.1 & 0.1 & -1.0 & -1.9 & -1.1 & $-3.1^{*}$ & -0.7 & -0.7 & $-2.5^{*}$ \\
\hline Low-fiber bread ${ }^{4}$ & $-4.2^{*}$ & $-4.8^{*}$ & $-3.8^{*}$ & $-6.1^{*}$ & $-5.4^{*}$ & $-4.3^{*}$ & $-3.4^{*}$ & $-3.5^{*}$ & -1.9 & $-3.9^{*}$ & $-5.5^{*}$ & $-4.6^{*}$ \\
\hline \multicolumn{13}{|l|}{ Unhealthy foods with higher intake in the US } \\
\hline Sodas, regular & 1.0 & 0.8 & 0.4 & $2.4^{*}$ & $2.8^{*}$ & $3.2^{*}$ & $3.1^{*}$ & $3.9^{*}$ & $4.2^{*}$ & $2.2^{*}$ & $3.3^{*}$ & $3.1^{*}$ \\
\hline High-fat cheeses ${ }^{3}$ & -0.1 & $1.1^{*}$ & $1.9^{*}$ & 0.6 & $1.4^{*}$ & $1.5^{*}$ & 0.9 & $1.6^{*}$ & $2.3^{*}$ & $0.9^{*}$ & $1.6^{*}$ & $1.9^{*}$ \\
\hline Desserts and salty snacks & $7.3^{*}$ & $9.0^{*}$ & $14^{*}$ & $7.4^{*}$ & $9.7^{*}$ & $15^{*}$ & $6.7^{*}$ & $7.0^{*}$ & $11^{*}$ & $5.7^{*}$ & $11^{*}$ & $13^{*}$ \\
\hline Pizzas and French fries & $2.9^{*}$ & $5.0^{*}$ & $5.7^{*}$ & $5.0^{*}$ & $7.6^{*}$ & $8.2^{*}$ & $5.5^{*}$ & $8.0^{*}$ & $8.1^{*}$ & $3.5^{*}$ & $5.9^{*}$ & $5.6^{*}$ \\
\hline \multicolumn{13}{|l|}{ Other effects of food acculturation 5} \\
\hline Ready-to-eat cereals & $2.6^{*}$ & $2.7^{*}$ & $2.6^{*}$ & $2.4^{*}$ & $2.3^{*}$ & $2.3^{*}$ & $2.4^{*}$ & $2.7^{*}$ & $2.7^{*}$ & 0.4 & 0.5 & $1.1^{*}$ \\
\hline Corn tortillas & $-13^{*}$ & $-16^{*}$ & $-17^{*}$ & $-18^{*}$ & $-21^{*}$ & $-22^{*}$ & $-22^{*}$ & $-25^{*}$ & $-26^{*}$ & $-18^{*}$ & $-24^{*}$ & $-25^{*}$ \\
\hline Wheat tortillas & -0.1 & -0.2 & $-1.1^{*}$ & 0.3 & -0.4 & $-1.6^{*}$ & $2.0^{*}$ & $0.9^{*}$ & -0.2 & $1.7^{*}$ & $1.8^{*}$ & $-0.8^{*}$ \\
\hline Atole & -0.3 & $-0.4^{*}$ & $-0.6^{*}$ & -0.4 & $-0.5^{*}$ & $-0.5^{*}$ & $-0.9^{*}$ & $-1.1^{*}$ & $-1.1^{*}$ & $-0.5^{*}$ & $-0.7^{*}$ & $-0.8^{*}$ \\
\hline Fruit juices & $2.7^{*}$ & $3.5^{*}$ & $3.6^{*}$ & $3.0^{*}$ & $2.0^{*}$ & $1.2^{*}$ & $2.9^{*}$ & $1.7^{*}$ & $1.5^{*}$ & $2.2^{*}$ & $0.8^{*}$ & $0.7^{*}$ \\
\hline
\end{tabular}

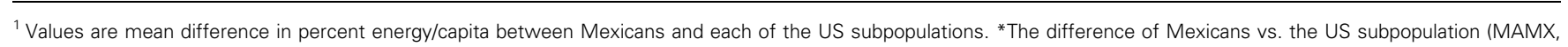

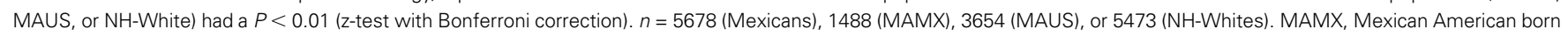
in Mexico; MAUS, Mexican American born in the US; NH-White, non-Hispanic white; MNS, Mexican Nutrition Survey.

${ }^{2}$ Low: $<2 \mathrm{~g}$ of fat/100 $\mathrm{g}$ of product; high: $\geq 2 \mathrm{~g} / 100 \mathrm{~g}$.

${ }^{3}$ Low: $\leq 10 \mathrm{~g}$ of fat/100 g of product; medium: $>10-20 \mathrm{~g} / 100 \mathrm{~g}$; high: $>20 \mathrm{~g} / 100 \mathrm{~g}$.

${ }^{4}$ Low: $\leq 2.5 \mathrm{~g}$ of fiber $/ 100 \mathrm{~g}$ or product; high: $>2.5 \mathrm{~g} / 100 \mathrm{~g}$.

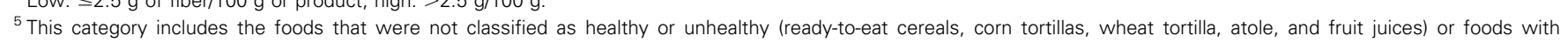
inconsistent results across groups (fruits and vegetables).

diet. This was especially true for the diets of the MAUS subpopulation, which showed very few significant differences from that of the NH-White subpopulation. Moreover, the differences seemed to be even fewer among children and female adolescents. Also, relative to the differences between the MAMX and the Mexican diet, the differences between MAMX and MAUS seemed small; perhaps a stratification of MAMX by length of time in the US could enlarge the differences in this study.

To the best of our knowledge, no previous study has simultaneously compared Mexican and MA diets with the objective of assessing food acculturation. One earlier study examined household food insecurity among 5-y-old children of Mexican descent living in migrant communities in California and Mexico. The authors did not directly compare the Mexican and Californian children's diets due to slight differences in the FFQ used in each country. Nevertheless, that study notes a more frequent intake of fruits, dairy, sweetened beverages, and fast foods and a less frequent intake of tortillas among children living in California compared to those living in Mexico (29). Although we did not use the exact same food groups or dietary measures, our results are in general consistent with this previous finding.
Numerous studies have compared the diets of Hispanics with different degrees of acculturation $(30,31)$. Some of the studies that used place of birth as a proxy for acculturation to compare the diets of populations of Mexican descent reported results similar to ours for the common food groups studied. In addition to fruits and vegetables and fruit and vegetable juices mentioned above, previous studies agreed that Mexican-born participants exhibited higher intakes of corn tortillas $(15,26)$, legumes $(8,14,15,26)$, and high-fat milk $(8,14)$ and lower intakes of salty snacks $(14,15)$, desserts $(14)$, fast food $(8,14)$, and diet sodas (26) compared to US-born participants.

The higher overall percentage of energy per capita coming from unhealthy foods found among MAUS compared to MAMX might help explain why some studies have found higher $\mathrm{BMI}$ in the more acculturated MA than in those less acculturated (6-8). However, it is well known that NH-Whites have a lower prevalence of obesity compared to MA (9) and in this study we also found that the intake of unhealthy foods was similar or slightly higher among NH-Whites than among MA. This supports that the cause of obesity among MA is complex and that other factors might interact with the diet. 


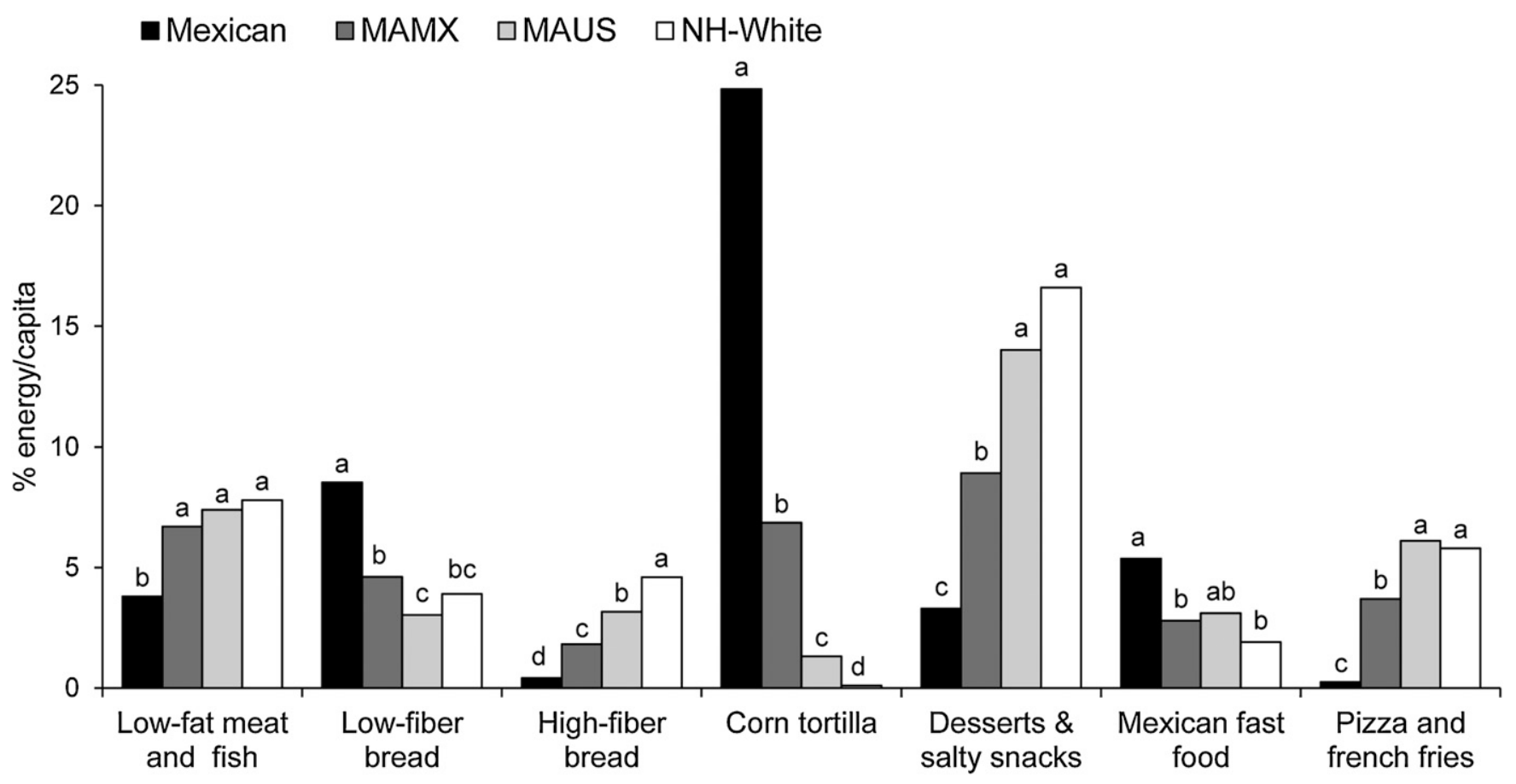

FIGURE 2 Percentage energy per capita from food groups with a difference of at least $\geq 4 \%$ among any 2 subpopulations in Mexican (MNS 1999), MA, and NH-White (NHANES 1999-2006) adult women. $n=1978$ (Mexican), 802 (MAMX), 486 (MAUS), or 2297 (NH-White). Percentages without a common letter differ, $P<0.01$ ( $z$-test with Bonferroni correction). MA, Mexican American; MAMX, Mexican American born in Mexico; MAUS, Mexican American born in the US; MNS, Mexican Nutrition Survey; NH-White, non-Hispanic white.

Although the current study provided good insight regarding how the country of birth influences food acculturation in MA populations, we need to keep in mind that these results described a scenario representative of roughly a decade ago. Unfortunately, for our aims, the MNS 1999 used here is the most recent nationally representative survey in Mexico that collected detailed

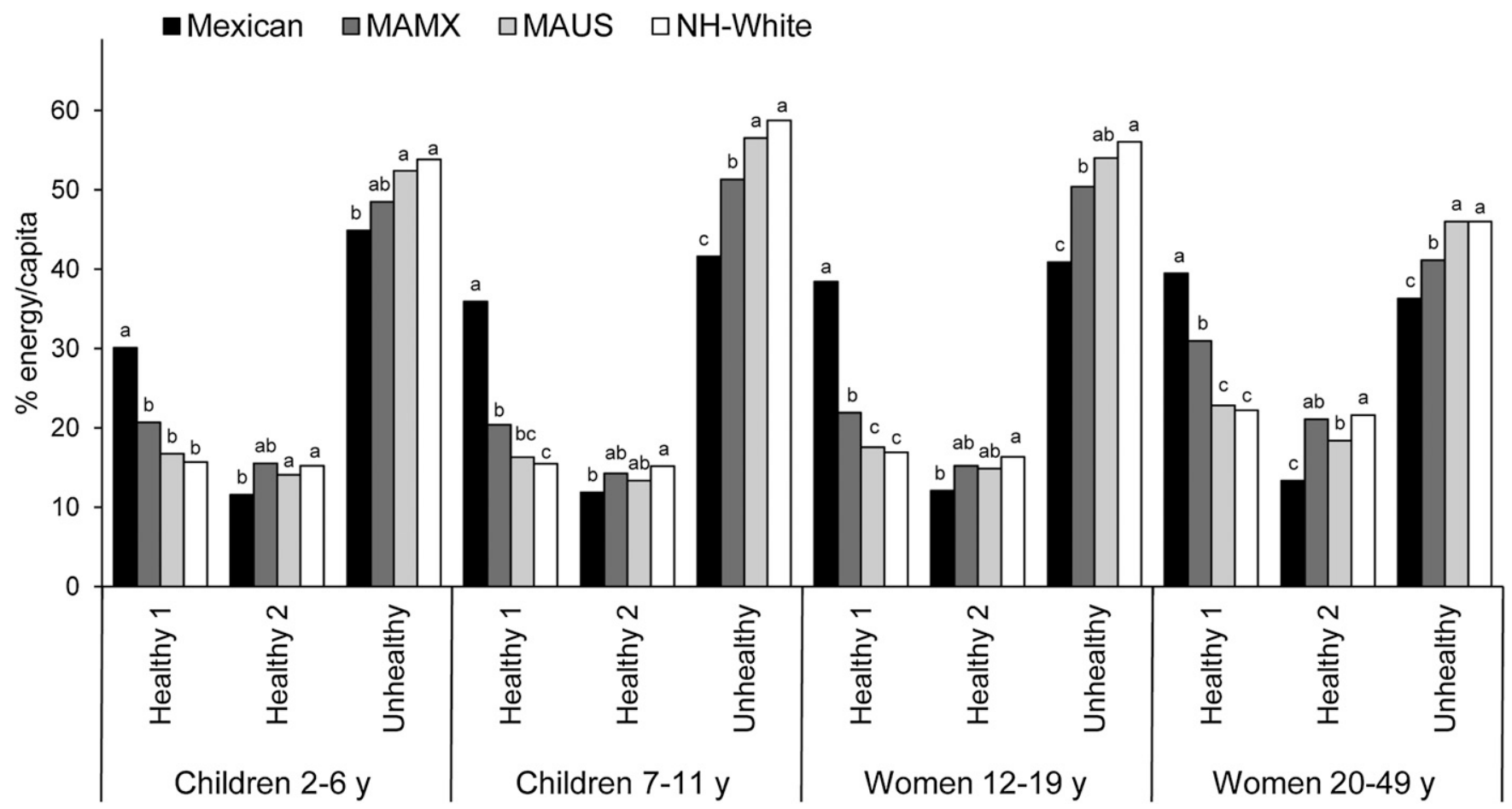

FIGURE 3 Mean percentage of total energy per capita from selected food groups aggregated into healthy or unhealthy items in Mexican (MNS 1999), MA, and NH-White (NHANES 1999-2006). Healthy 1 includes low-fat milk, low-fat meat and fish, beans and other legumes, nuts and seeds, high-fiber bread, tortillas (corn and wheat), and fruits and vegetables. Healthy 2 includes the same items as Healthy 1 but excludes tortillas. Unhealthy includes regular soda, high-fat milk, medium- and high-fat meat, low-fiber bread, desserts, salty snacks, Mexican fast food, pizza, and French fries. $n=5678$ (Mexicans), 1488 (MAMX), 3654 (MAUS), or 5473 (NH-Whites). Percentages without a common letter differ, $P<0.01$ (z-test with Bonferroni correction). MA, Mexican American; MAMX, Mexican American born in Mexico; MAUS, Mexican American born in the US; MNS, Mexican Nutrition Survey; NH-White, non-Hispanic white. 
data with a 24-h dietary recall instrument comparable to that used by NHANES. A more recent national survey in Mexico in 2006 (32) used a 1-wk FFQ and therefore was not comparable to the methodology used in NHANES. Many ongoing changes in both Mexico and the US might affect the differences among the subpopulations in this study. For one, Mexico is undergoing a nutrition transition. A significant increase in energy from beverages was reported in Mexican preschoolers and school-age children between 1999 and 2006 (13). Energy from soft drinks, sweetened juices, and sugared coffee more than doubled in adolescents between 1999 and 2006 and it tripled in adult women in that time period (33). Based on the Mexican Household Income and Expenditure Surveys, the percentage of Mexican households purchasing soda increased from $57 \%$ in 1998 to $61 \%$ in 2006 (33). In addition, the intake of corn tortillas by Mexicans decreased from $275 \mathrm{~g} /$ capita in 2000 to between 180 and $190 \mathrm{~g}$ in 2009 (34). Based on these data, we could hypothesize that Mexicans, due to their country's geographical proximity and highly influenced relationship with the US, could be approximating the dietary profiles of the MA described in this study.

Changes in the US may also influence the food acculturation process. It has been suggested that, in areas of high Latino density, acculturation could be expected to occur in the opposite direction; i.e. non-Hispanic Americans are becoming acculturated into the Latino culture (31). Modifications in the US food supply and increased availability of Mexican ingredients and foods might also help MA preserve their original diets. The differences among NH-Whites, MA, and Mexican diets that exist now and in the near future can only be explored as new adequate data become available.

Our results should be viewed with caution in the sense that we compared data obtained from 2 different surveys. Even though both countries used 24-h dietary recalls, some evident and nonevident survey differences might affect the results. An important evident difference was that, in Mexico, most of the intake was reported by ingredient, whereas in the US, a larger proportion was reported as the final prepared food. Although we made an effort to combine the ingredients of key foods into a single food group, like beverages, and to report only food groups that were captured well by both surveys, we still might have missed some crucial information. Moreover, because the 24-h methodology in Mexico did not include a 5-pass or similar probing method, we cannot reject the possibility that the degree of underreporting or differential reporting of healthy or unhealthy foods was different in the 2 surveys.

Another limitation of this study is that it does not include information on male adolescents and adults or on older adults of either gender. This fact severely limits the general picture we can get of how food acculturation affects the diets of all age and gender groups.

We also acknowledge that acculturation is a complex phenomenon that cannot be quantified by a simple static proxy indicator like birthplace. As has been suggested, the acculturation process is complex and multidimensional. Hispanics may become assimilated, integrated, segregated, or marginalized to the American mainstream culture (31). Our intention in this study was therefore to use birthplace as a proxy of exposure to the US environment rather than an overall measure of the degree of acculturation. We think that by assessing the Mexican and US influences on the diets of MA born in Mexico and those born in the US, we are, indeed, approaching a better understanding of the food acculturation process of these subpopulations with different degrees of exposure to the US environment.
In sum, our results indicate that Mexicans in 1999 appeared to have eaten a healthier diet than US subpopulations between 1999 and 2006 and that, as the level of exposure to the US environment increased, the MA diet shifted toward an unhealthier one. MAMX who had adopted many aspects of the US diet but still preserved many from their original Mexican culture appeared to have healthier diets than MAUS. In addition, the differences between the MAUS and NH-White subpopulations were minimal, suggesting that the food acculturation process might be almost completed within one generation among the MA population. These results strengthen our understanding of acculturation to the American food environment among MA and they reinforce the need to discourage critical unhealthful components of the American diet among MA.

\section{Acknowledgments}

We thank Dr. Phil Bardsley and Rick O'Hara for exceptional assistance with the data management and programming, Frances L. Dancy for administrative assistance, and Tom Swasey for graphics support. We also thank Carmen Piernas, Jennifer M. Poti, and Susan Kleiman for their helpful comments on this manuscript. C.B., S.B., J.A.R, and B.M.P. designed research; C.B. analyzed data; C.B. and B.M.P. wrote the paper; L.H.B, S.B., and J.A.R provided databases; and B.M.P. had primary responsibility for final content. All authors read and approved the final manuscript.

\section{Literature Cited}

1. Humes KR, Jones NA, Ramirez RR. Overview of race and Hispanic origin: 2010: US Census Bureau; 2011.

2. Ramirez RR, de la Cruz GP. The Hispanic population in the United States: March 2002. Washington, DC: US Census Bureau; 2002.

3. Day JC. Population projections of the United States by age, sex, race, and Hispanic origin: 1995 to 2050. Washington, DC: Government Printing Office, US Bureau of the Census; 1996.

4. Morales LS, Lara M, Kington RS, Valdez RO, Escarce JJ. Socioeconomic, cultural, and behavioral factors affecting Hispanic health outcomes. J Health Care Poor Underserved. 2002;13:477-503.

5. Barcenas CH, Wilkinson AV, Strom SS, Cao Y, Saunders KC, Mahabir S, Hernandez-Valero MA, Forman MR, Spitz MR, Bondy ML. Birthplace, years of residence in the United States, and obesity among MexicanAmerican adults. Obesity (Silver Spring). 2007;15:1043-52.

6. Yeh MC, Viladrich A, Bruning N, Roye C. Determinants of Latina obesity in the United States: the role of selective acculturation. J Transcult Nurs. 2009;20:105-15.

7. Popkin BM, Udry JR. Adolescent obesity increases significantly in second and third generation US immigrants: the National Longitudinal Study of Adolescent Health. J Nutr. 1998;128:701-6.

8. Gordon-Larsen P, Harris KM, Ward DS, Popkin BM. Acculturation and overweight-related behaviors among Hispanic immigrants to the US: the National Longitudinal Study of Adolescent Health. Soc Sci Med. 2003;57:2023-34.

9. Flegal KM, Carroll MD, Ogden CL, Curtin LR. Prevalence and trends in obesity among US adults, 1999-2008. JAMA. 2010;303:235-41.

10. Rivera JA, Barquera S, Gonzalez-Cossio T, Olaiz G, Sepulveda J. Nutrition transition in Mexico and in other Latin American countries. Nutr Rev. 2004;62:S149-57.

11. Durazo-Arvizu R, Barquera S, Franco M, Lazo M, Seuc A, Orduñez P, Palloni A, Cooper RS. Cardiovascular diseases mortality in Cuba, Mexico, Puerto Rico and US Hispanic populations. Prev Control. 2006;2:63-71.

12. Villalpando S, Carrión C, Barquera S, Olaiz-Fernández G, Robledo R. Body mass index associated with hyperglycemia and alterations of components of metabolic syndrome in Mexican adolescents. Salud Publica Mex [Internet]. 2007 [cited 2010 Dec 18];49:s324-30. Available from: http://www.scielo.org.mx/scielo.php?script=sci_arttext\&pid=S003636342007000900003\&lng=es\&nrm=iso. 2007;49:s324-30. 
13. Barquera S, Campirano F, Bonvecchio A, Hernández L, Rivera J, Popkin B. Caloric beverage consumption patterns in Mexican children. Nutr J. 2010;9:47-56.

14. Duffey KJ, Gordon-Larsen P, Ayala GX, Popkin BM. Birthplace is associated with more adverse dietary profiles for US-born than for foreign-born Latino adults. J Nutr. 2008;138:2428-35.

15. Dixon LB, Sundquist J, Winkleby M. Differences in energy, nutrient, and food intakes in a US sample of Mexican-American women and men: findings from the Third National Health and Nutrition Examination Survey, 1988-1994. Am J Epidemiol. 2000;152:548-57.

16. Aldrich L, Variyam JN. Acculturation erodes the diet quality of US Hispanics. Food Review. 2000;23:51-5.

17. Guendelman S, Abrams B. Dietary intake among Mexican-American women: generational differences and a comparison with white nonHispanic women. Am J Public Health. 1995;85:20-5.

18. Rivera-Dommarco J, Shamah-Levy T, Villalpando-Hernandez S, GonzalezCossio T, Hernandez-Prado B, Sepulveda J. Encuesta Nacional de Nutricion 1999. Estado nutricio de niños y mujeres en México. Cuernavaca, Morelos (México): Instituto Nacional de Salud Pública; 2001.

19. National Center for Health Statistics. NHANES analytic and reporting guidelines. 2006 [cited 2011 Jan 27]. Available from: http://www.cdc. gov/nchs/data/nhanes/nhanes_03_04/nhanes_analytic_guidelines_dec_ 2005.pdf.

20. Dwyer J, Picciano MF, Raiten DJ, Members of the Steering Committee, National Health and Nutrition Examination Survey. Collection of food and dietary supplement intake data: What We Eat in AmericaNHANES. J Nutr. 2003;133:S590-600.

21. CDC National Center for Health Statistics. National Health and Nutrition Examination Survey: dietary interviewers procedures manual; 2000 [cited 2010 Nov 29]. Available from: http://www.cdc.gov/nchs/ data/nhanes/dr-1-5.pdf.

22. Wright JD, Borrud LG, McDowell MA, Wang CY, Radimer K, Johnson CL. Nutrition assessment in the National Health And Nutrition Examination Survey 1999-2002. J Am Diet Assoc. 2007;107:822-9.

23. Popkin BM, Haines P, Siega-Riz A. Dietary patterns and trends in the United States: the UNC-CH approach. Appetite. 1999;32:8-14.
24. Altman DG, Bland JM. Interaction revisited: the difference between two estimates. BMJ. 2003;326:219.

25. Kuczmarski RJ, Ogden CL, Grummer-Strawn LM, Flegal KM, Guo SS, Wei R, Mei Z, Curtin LR, Roche AF, Johnson CL. CDC growth charts: United States. Adv Data. 2000;1-27.

26. Monroe KR, Hankin JH, Pike MC, Henderson BE, Stram DO, Park S, Nomura AM, Wilkens LR, Kolonel LN. Correlation of dietary intake and colorectal cancer incidence among Mexican-American migrants: the multiethnic cohort study. Nutr Cancer. 2003;45:133-47.

27. Sharma S, Murphy SP, Wilkens LR, Shen L, Hankin JH, Monroe KR, Henderson B, Kolonel LN. Adherence to the food guide pyramid recommendations among African Americans and Latinos: results from the Multiethnic Cohort. J Am Diet Assoc. 2004;104:1873-7.

28. Romero-Gwynn E, Gwynn D, Grivetti L, McDonald R, Stanford G, Turner B, West E, Williamson E. Dietary acculturation among Latinos of Mexican descent. Nutr Today. 1993;28:6-12.

29. Rosas LG, Harley K, Fernald LC, Guendelman S, Mejia F, Neufeld LM, Eskenazi B. Dietary associations of household food insecurity among children of Mexican descent: results of a binational study. J Am Diet Assoc. 2009;109:2001-9.

30. Ayala GX, Baquero B, Klinger S. A systematic review of the relationship between acculturation and diet among Latinos in the United States: implications for future research. J Am Diet Assoc. 2008;108:1330-44.

31. Perez-Escamilla R, Putnik P. The role of acculturation in nutrition, lifestyle, and incidence of type 2 diabetes among Latinos. J Nutr. 2007;137:860-70.

32. Olaiz G, Rivera J, Shamah T, Rojas R, Villalpando S, Hernandez M, Sepulveda J. Encuesta Nacional de Salud y Nutricion 2006. Cuernava (Mexico): Instituto Nacional de Salud Publica; 2006.

33. Barquera S, Hernandez-Barrera L, Tolentino ML, Espinosa J, Ng SW, Rivera JA, Popkin BM. Energy intake from beverages is increasing among Mexican adolescents and adults. J Nutr. 2008;138:2454-61.

34. Leon-Zaragoza G. Cae consumo de tortilla ya no se compra por kilo. La Jornada. 2009 March 17; Sect. Politica. p.16. 